\title{
Treatment of chronic inflammatory diseases with implantable medical devices*
}

\begin{abstract}
Implantable medical devices are finding increasing use in the treatment of diseases traditionally targeted with drugs. It is well established that the cholinergic antiinflammatory pathway serves as a physiological regulator of inflammatory responses, but stimulation of this pathway therapeutically by electrical stimulation of the vagus nerve can also diminish excessive or dysregulated states of inflammation. Recent data from a wide variety of animal models, as well as evidence of reduced vagal tone in rheumatoid arthritis and other inflammatory diseases, support the rationale for, and feasibility of, developing implantable vagal nerve stimulation devices to treat chronic inflammation in humans.
\end{abstract}

mplantable devices are increasingly used in the treatment of diseases which have historically been targeted only with small molecule and biological therapeutic agents. In addition to wellestablished products such as subcutaneous insulin pumps and intra-arterial chemotherapy pumps, where the implantable device merely serves as a more efficient means of delivering the drug, there are a number of recently developed therapeutic approaches in which the implanted device itself functions to directly treat the underlying medical condition. One particularly successful example of this strategy is cardiac resynchronization using biventricular pacing devices for congestive heart failure (CHF). These devices were approved for marketing after having been proved to prolong survival in patients whose disease had progressed despite medical management. ${ }^{1}$ Implantable device products are now approved or in late-stage development for many other traditional

\footnotetext{
* This article is reprinted from Annals of the Rheumatic Diseases (Zitnik RJ. Treatment of chronic inflammatory diseases with implantable medical devices. Ann Rheum Dis 2011; 70[suppl 1]:i67-i70) with permission from the publisher.

Dr. Zitnik reported that he is employed by and has intellectual property rights and ownership interest in SetPoint Medical.

doi:10.3949/ccjm.78.s1.05
}

"medical" disorders such as hypertension, obesity, diabetes, Parkinson's disease, and glaucoma. Recent advances in understanding the interplay between the central nervous system and the immune system have made possible a feasible implantable device approach that may similarly find use in the management of rheumatoid arthritis (RA) and other chronic inflammatory diseases. ${ }^{2}$

\section{NEUROSTIMULATION OF THE CHOLINERGIC ANTIINFLAMMATORY PATHWAY}

The vagus nerve mediates an important neural reflex which senses inflammation both peripherally and in the central nervous system, and downregulates the inflammation via efferent neural outflow to the reticuloendothelial system. The efferent arm of this reflex has been termed the "cholinergic antiinflammatory pathway" (CAP). The CAP serves as a physiological regulator of inflammation by responding to environmental injury, pathogens, and other external threats with an appropriate degree of immune system activation. ${ }^{3}$ An increasing body of evidence indicates that the CAP can also be harnessed to reduce pathological inflammation. Electrical neurostimulation of the vagus nerve (NCAP) in an appropriate manner with an implantable device is emerging as a novel and potentially feasible means of treating diseases characterized by excessive and dysregulated inflammation.

Our current understanding of the CAP began with the observations of Kevin Tracey and colleagues over a decade ago. They demonstrated that systemic, hepatic, and splenic tumor necrosis factor (TNF) production as well as the physiological manifestations of endotoxemic shock in rodents were worsened by vagotomy and ameliorated by electrical stimulation of the cervical vagus nerve (VNS). Further, based on in vitro experiments they postulated that this effect was mediated directly by acetylcholine acting through specific receptors on macrophages in the reticuloendothelial system. ${ }^{4}$ It was later demonstrated that reducing the response to endotoxemia 
TABLE 1

CAP stimulation favourably affects many components of the immune system

\begin{abstract}
Immune system component
Monocytes-macrophages

Neutrophils

T cells

Effect

VNS or pharmacological manipulation of the CAP causes reduced production of:

$\rightarrow \mathrm{TNF} \alpha$

- IL-1 $\beta$

- IL-6

IFNy

HMGB1

- CXCL-2

And no change or increased production of

- TGF $\beta$

- IL-10

VNS or pharmacological manipulation of the CAP causes reduced cellular trafficking to sites of inflammation, driven by reduction in cell surface CD11b expression

Vagotomy reduces T regulatory cells (CD4(+)FoxP3(+)), and long-term recovery from the immediate proinflammatory effects of vagotomy is associated with return of T regulatory cells Proinflammatory effect of vagotomy can be adoptively transferred Vagotomy increases and pharmacological CAP agonists decrease CD4(+)CD25(-) proliferation and production of II-6, IFN $\gamma$ and TNF
\end{abstract}

HPA axis

VNS increases systemic corticosteroid production via vagal afferent pathways

$\mathrm{CAP}=$ cholinergic anti-inflammatory pathway; $\mathrm{HMGB}=$ high-mobility group box 1 ; IFN = interferon; IL = interleukin; TGF = transforming growth factor; $\mathrm{TNF}=$ tumor necrosis factor; VNS = vagus nerve stimulation

using NCAP required an intact spleen, and selective anatomical lesion experiments showed that an intact neural pathway to the spleen from the cervical vagus through the celiac ganglion and the splenic nerve was also necessary for this effect. ${ }^{5}$ Within the spleen itself, nerve fiber synaptic vesicles are found in close apposition to TNF-secreting macrophages. ${ }^{6}$ The $\alpha-7$ nicotinic acetylcholine receptor, expressed on the surface of macrophages, is essential for the NCAP effect as demonstrated by antisense oligonucleotide and targeted disruption experiments. ${ }^{7}$ In the macrophage, the $\alpha-7$ nicotinic acetylcholine receptor does not appear to transduce signals through ion channels, as is the case in neuronal tissue. Rather, the NCAP effect is mediated at the subcellular level by alterations in the NF- $\mathrm{kB}$ and JAK/STAT/SOCS pathways. ${ }^{8,9}$

In addition to effects on macrophages, CAP stimulation has more recently been shown to alter the function of other components of the cellular immune system. The trafficking of neutrophils to sites of inflammation is reduced by VNS, accompanied by reductions in $\mathrm{CD} 11 \mathrm{~b}$ expression. ${ }^{9,10}$ In rodent colitis models, disease severity is worsened by vagotomy, which simultaneously reduces the number of circulating Foxp $3+\mathrm{T}$ regulatory cells. The proinflammatory effect of vagotomy can be adoptively transferred with a CD4+CD25-T-cell subpopulation. ${ }^{11}$ Over time the proinflammatory effect of vagotomy wanes, accompanied by recovery of $\mathrm{T}$ regulatory cell numbers. ${ }^{12,13}$ Finally, vagotomy and pharmacological manipulation of the CAP alter in vitro T-cell proliferation and production of the Th1 cytokines interferon $\gamma$, TNF, and interleukin 6 (Table 1). ${ }^{14}$

When taken together, these studies show that NCAP has a dual set of immunological effects: it reduces production of systemically active cytokines by resident spleen cells and also causes circulating cells which traverse the spleen to develop an altered phenotype with reduced expression of inflammatory mediators and adhesion molecules upon trafficking to inflamed tissue.

An important characteristic of NCAP delivered by VNS is that very brief episodes of stimulation result in a remarkably prolonged biological effect. Huston et al delivered a single 30 -second electrical VNS or sham treatment in rats, and then induced endotoxemia with intraperitoneal lipopolysaccharide (LPS) at varying times after VNS. Interestingly, this brief VNS stimulation reduced production of serum TNF in response to systemic LPS exposure for up to 48 hours. Similarly, after only 60 minutes of exposure to acetylcholine, cul- 
TABLE 2

Efficacy of neurostimulation of the cholinergic anti-inflammatory pathway in animal models

Acute models

Endotoxemia and sepsis

Cecal puncture/acute peritonitis

Ischemia-reperfusion

Acute pancreatitis

Myocardial infarction

Subacute and chronic models

Inflammatory postoperative ileus

Congestive heart failure

Collagen-induced arthritis

tured human macrophages are changed in phenotype such that they become refractory to in vitro LPS stimulation for up to 48 hours thereafter. ${ }^{15}$ The consistency of this phenomenon across species is corroborated by preliminary data in a canine model where 60 -second VNS treatment results in reduced LPS-inducible TNF production in a whole-blood in vitro release assay for several days after the VNS (M Faltys, personal communication). A duration of biological effect lasting hours to days after periods of stimulation lasting for only seconds to minutes implies that an implantable device will probably only need to operate with very short daily duty cycles to effectively elicit an NCAP response. This will in turn greatly reduce the necessary size and complexity of the device itself, and increase its functional lifespan, with resultant reductions in overall cost of the treatment.

\section{NEUROSTIMULATION OF THE CAP IN ANIMAL MODELS OF DISEASE}

CAP stimulation, delivered either by electrical VNS or by pharmacological agonists has proved quite effective in a wide variety of acute disease models of inflammation, including pancreatitis, myocardial infarction, ischemia-reperfusion injury, acute peritonitis, and hemorrhagic shock (Table 2) (reviewed by Tracey $^{3}$ ). Until recently, testing for effectiveness of NCAP in more subacute and chronic rodent models of inflammation was not possible as the ability to deliver VNS was limited to "single sitting" surgical sessions because no reliable chronic rodent VNS system was available. However, a chronically implantable exter-

nalized lead with an external pulse generator, suitable for use in rodents, has now been developed (Figure). This system allows testing of NCAP in a variety of standard inflammation models. In preliminary data presented in abstract form, NCAP has been shown to be effective in reducing clinical signs and histological joint damage in a rat collagen-induced arthritis model, even though active VNS was only delivered for 60 seconds daily. ${ }^{16}$ Owing to the prohibitively large size of the current prototype rodent vagus nerve lead, at present it can only be used in rats and similarly sized animals. Future iterations of this delivery system will be smaller, self-contained, and fully implantable, allowing use over several months in more chronic models, and implantation in mice, both of which will greatly expand its usefulness as a research tool.

In a canine model of CHF induced by rapid ventricular pacing, inflammation and ventricular remodeling with fibrosis are typically accompanied by marked increases in serum $\mathrm{C}$-reactive protein (CRP) levels. In addition to improving the physiological manifestations of CHF, VNS resulted in $60 \%$ to $80 \%$ reductions in CRP for up to 8 weeks. ${ }^{17}$ In another canine CHF model induced by repetitive microembolization, which is similarly associated with systemic and myocardial inflammation, VNS markedly reduced circulating levels of interleukin 6 and TNF for up to 12 weeks. ${ }^{18}$ Importantly, both these studies show that rapid tachyphylaxis does not appear to occur with NCAP over periods of time that are relatively chronic by the typical standards of animal models.

\section{VAGAL NERVE STIMULATION FOR EPILEPSY AND DEPRESSION: EXPERIENCE IN HUMANS}

VNS delivered using a surgically implanted cuffed cervical vagus nerve lead and pacemaker-style pulse generator device has been approved for the treatment of refractory epilepsy in the United States since the mid-1990s and has more recently been approved for treatment of depression. Over 50,000 patients have been implanted with these devices world wide since that time. The safety profile of both surgical implantation and VNS delivery in this setting is well established. ${ }^{19}$ The major tolerability problem is laryngeal and pharyngeal symptoms, such as hoarseness and dysphonia, which are present almost solely during periods of active device stimulation. The frequency and severity of these treatments decreases after receiving treatment for an extended time. ${ }^{20}$ With growing experience in VNS delivery over the first 5 years of use, it also became apparent that reducing the active 
stimulation duty cycle from $40 \%$ to $10 \%$, and keeping stimulation currents at $\leq 1.5 \mathrm{~mA}$ results in a marked reduction in these symptoms. ${ }^{21}$ Of note, the stimulation currents necessary to evoke NCAP in animals are well below the $1.5 \mathrm{~mA}$ level, and as above, NCAP is effective even with very brief, once-daily periods of stimulation (ie, a duty cycle of $0.07 \%$ if given for $1 \mathrm{~min}$ each day). Thus it is likely that the laryngeal and pharyngeal adverse event profile of VNS will not be problematic in the setting of NCAP delivery for inflammation.

\section{A POTENTIAL ROLE FOR THERAPEUTIC NCAP USING IMPLANTABLE DEVICES IN HUMAN INFLAMMATORY DISEASES}

Autonomic nervous system activity can be measured indirectly by recording cardiac R-R interval variability and subjecting the data to power spectral analysis. Such heart rate variability (HRV) measurements are influenced by the levels of vagus nerve activity and by balance in cardiac sympathetic-parasympathetic tone. Reduced HRV is indicative of decreased vagal tone, and reductions in HRV have a strong inverse correlation with CRP levels, progression of atherosclerosis, and risk of sudden death. ${ }^{22,23}$ HRV is also reduced relative to normal subjects in patients with RA, systemic lupus erythematosus, and Sjögren syndrome, and the extent of reduction in HRV within the patient groups correlates with disease severity. ${ }^{24-26}$ Although these associations are only correlative and do not provide firm evidence of causality, they do provide additional epidemiological support for the hypothesis that driving increased vagal activity using implantable devices may have a favorable effect on inflammatory disease.

Implantable neurostimulation devices have not yet been tested in human patients with RA. However, preliminary evidence from a small study carried out in normal volunteers demonstrated that the CAP reflex can be elicited by brief mechanical stimulation of the afferent auricular branch of the vagus nerve, as shown by reduction of in vitro LPS-inducible cytokine production (T van der Poll, personal communication). Clinical testing of NCAP using implantable VNS devices will begin in the near future. The devices to be used for these initial studies will be very similar in design to those currently in use for epilepsy treatment. However, prototype versions of the device which will be used in follow-on studies are miniaturized to the point where they will be directly implantable on the vagus nerve, without the need for a pulse generator unit on the chest and will

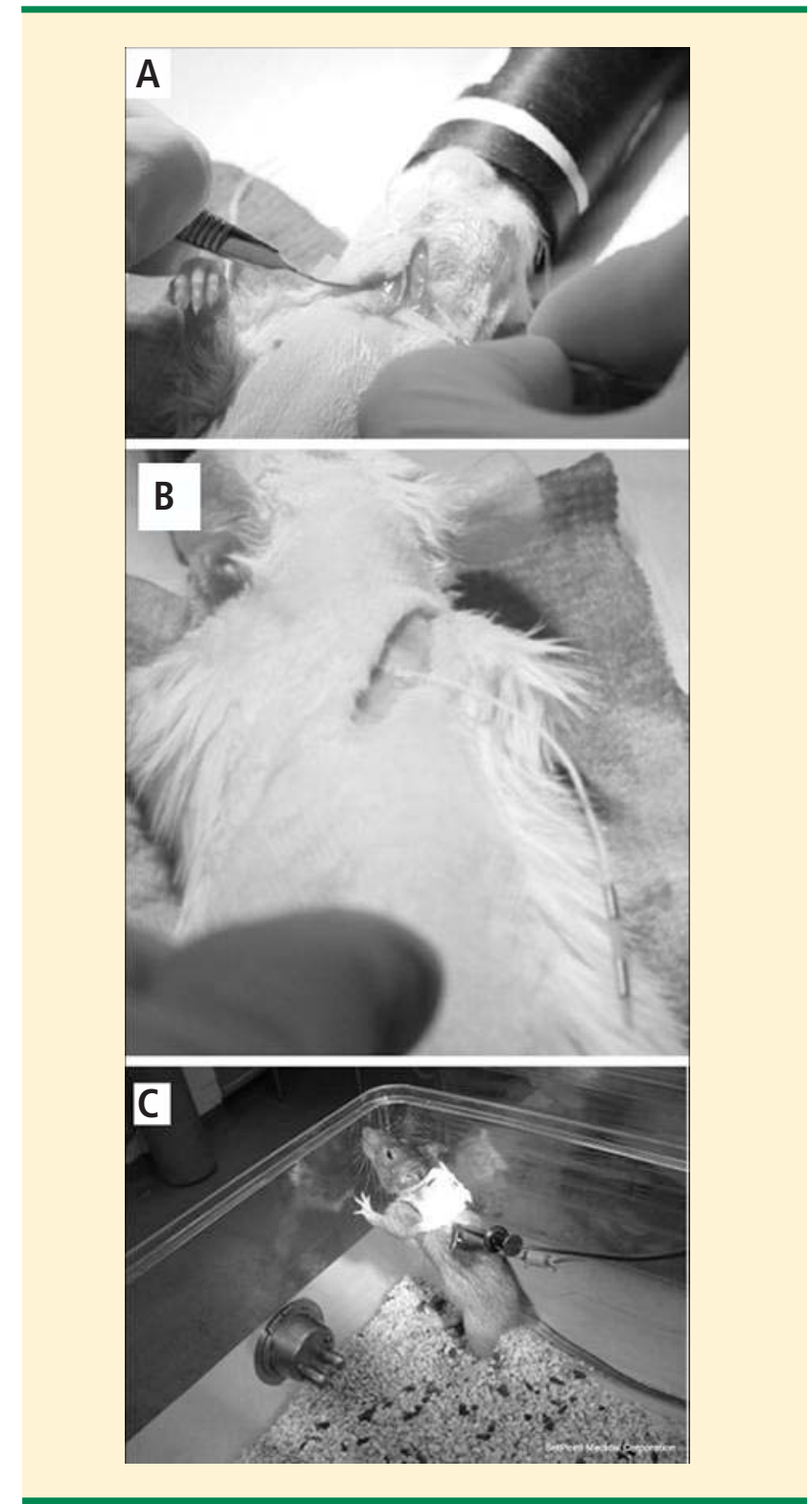

FIGURE. An implantable lead system for delivering neurostimulation of the cholinergic anti-inflammatory pathway (NCAP) in rodents. (A) Lead is wrapped circumferentially around left carotid sheath. (B) After anterior closure, lead is tunnelled subcutaneously and externalized posteriorly in the midline. (C) Animal is jacketed to prevent damage to externalized electrode. NCAP is delivered by temporarily connecting leads to an external electronic pulse generator.

use a small self-contained battery system which can be recharged using transcutaneous radiofrequency induction. Given the long lifespan, relatively low cost, and potential for increased safety over currently available treatments, NCAP delivered using an implantable device holds great promise as a novel potential therapeutic approach for patients with RA and other inflammatory diseases. 


\section{REFERENCES}

1. Bristow MR, Saxon LA, Boehmer J, et al. Comparison of Medical Therapy, Pacing, and Defibrillation in Heart Failure (COMPANION) Investigators. Cardiac-resynchronization therapy with or without an implantable defibrillator in advanced chronic heart failure. N Engl J Med 2004; 350:2140-2150.

2. van Maanen MA, Vervoordeldonk MJ, Tak PP. The cholinergic anti-inflammatory pathway: towards innovative treatment of rheumatoid arthritis. Nat Rev Rheumatol 2009; 5:229-232.

3. Tracey KJ. Reflex control of immunity. Nat Rev Immunol 2009; 9:418-428.

4. Borovikova LV, Ivanova S, Zhang M, et al. Vagus nerve stimulation attenuates the systemic inflammatory response to endotoxin. Nature 2000; 405:458-462.

5. Huston JM, Ochani M, Rosas-Ballina M, et al. Splenectomy inactivates the cholinergic antiinflammatory pathway during lethal endotoxemia and polymicrobial sepsis. J Exp Med 2006; 203:1623-1628.

6. Rosas-Ballina M, Ochani M, Parrish WR, et al. Splenic nerve is required for cholinergic antiinflammtory pathway control of TNF in endotoxemia. Proc Natl Acad Sci 2008; 105:11008-11013.

7. Wang $\mathrm{H}, \mathrm{Yu} \mathbf{M}$, Ochani $\mathbf{M}$, et al. Nicotinic acetylcholine receptor alpha7 subunit is an essential regulator of inflammation. Nature 2003; 421:384-388.

8. Wang $\mathrm{H}$, Liao $\mathrm{H}$, Ochani $\mathrm{M}$, et al. Cholinergic agonists inhibit HMGB1 release and improve survival in experimental sepsis. Nat Med 2004; 10:1216-1221.

9. de Jonge WJ, van der Zanden EP, The FO, et al. Stimulation of the vagus nerve attenuates macrophage activation by activating the Jak2-STAT3 signaling pathway. Nat Immunol 2005; 6:844-851.

10. Huston JM, Rosas-Ballina M, Xue X, et al. Cholinergic neural signals to the spleen down-regulate leukocyte trafficking via CD11b. J Immunol 2009; 183:552-559.

11. O’Mahony C, van der Kleij $\mathrm{H}$, Bienenstock J, et al. Loss of vagal anti-inflammatory effect: in vivo visualization and adoptive transfer. Am J Physiol Regul Integr Comp Physiol 2009; 297:R1118-R1126.

12. Ghia JE, Blennerhassett P, Kumar-Ondiveeran $\mathrm{H}$, et al. The vagus nerve: a tonic inhibitory influence associated with inflammatory bowel disease in a murine model. Gastroenterology 2006; 131:1122-1130.

13. Ghia JE, Blennerhassett P, Collins SM. Vagus nerve integrity and experimental colitis. Am J Physiol Gastrointest Liver Physiol 2007; 293:G560-G567.

14. Karimi K, Bienenstock J, Wang L, et al. The vagus nerve modu- lates CD4+ T cell activity. Brain Behav Immun 2010; 24:316-323.

15. Huston JM, Gallowitsch-Puerta M, Ochani M, et al. Transcutaneous vagus nerve stimulation reduces serum high mobility group box 1 levels and improves survival in murine sepsis. Crit Care Med 2007; 35:2762-2768.

16. Levine Y, Faltys M, Black K, et al. Neurostimulation of the cholinergic anti-inflammatory pathway (NCAP) ameliorates CIA in rats. Ann Rheum Dis 2010; 69(suppl 3):191.

17. Zhang Y, Popovic ZB, Bibevski S, et al. Chronic vagus nerve stimulation improves autonomic control and attenuates systemic inflammation and heart failure progression in a canine high-rate pacing model. Circ Heart Fail 2009; 2:692-699.

18. Gupta RC, Imai M, Jiang AJ, et al. Chronic therapy with selective vagus nerve stimulation normalizes plasma concentration of tumor necrosis factor alpha, interleukin-6, and B-type natriuretic peptide in dogs with heart failure. J Am Coll Cardiol 2006; 47:77A.

19. Beekwilder JP, Beems T. Overview of the clinical applications of vagus nerve stimulation. J Clin Neurophysiol 2010; 27:130-138.

20. Ben-Menachem E. Vagus nerve stimulation, side effects, and longterm safety. J Clin Neurophysiol 2001; 18:415-418.

21. Heck C, Helmers SL, DeGiorgio CM. Vagus nerve stimulation therapy, epilepsy, and device parameters: scientific basis and recommendations for use. Neurology 2002; 59(6 suppl 4):S31-S37.

22. Huikuri HV, Jokinen V, Syvänne M, et al. Heart rate variability and progression of coronary atherosclerosis. Arterioscler Thromb Vasc Biol 1999; 19:1979-1985.

23. Sajadieh A, Nielsen OW, Rasmussen V, et al. Increased heart rate and reduced heart-rate variability are associated with subclinical inflammation in middle-aged and elderly subjects with no apparent heart disease. Eur Heart J 2004; 25:363-370.

24. Louthrenoo W, Ruttanaumpawan P, Aramrattana A, et al. Cardiovascular autonomic nervous system dysfunction in patients with rheumatoid arthritis and systemic lupus erythematosus. QJM 1999; 92:97-102.

25. Evrengül H, Dursunoglu D, Cobankara V, et al. Heart rate variability in patients with rheumatoid arthritis. Rheumatol Int 2004; 24:198-202.

26. Stojanovich L, Milovanovich B, de Luka SR, et al. Cardiovascular autonomic dysfunction in systemic lupus, rheumatoid arthritis, primary Sjögren syndrome and other autoimmune diseases. Lupus 2007; 16:181-185.

Correspondence: Ralph J. Zitnik, MD, SetPoint Medical Corporation, 222 Berkeley Street, 20th Floor, Boston, MA 02116; rzitnik@setpointmedical.com 